\title{
Cerebrospinal fluid reconstitution via a perfusion-based cadaveric model: feasibility study demonstrating surgical simulation of neuroendoscopic procedures
}

\author{
Jesse L. Winer, MD, ${ }^{1}$ Daniel R. Kramer, MD, ${ }^{1}$ Richard A. Robison, MD, Ifije Ohiorhenuan, MD, PhD, ${ }^{1}$ \\ Michael Minneti, BS, RRT, ${ }^{2}$ Steven Giannotta, MD, ${ }^{1}$ and Gabriel Zada, MD1 \\ Departments of ${ }^{1}$ Neurosurgery and ${ }^{2}$ General Surgery, University of Southern California, Los Angeles, California
}

Cadaveric surgical simulation carries the advantage of realistic anatomy and haptic feedback but has been historically difficult to model for intraventricular approaches given the need for active flow of CSF. This feasibility study was designed to simulate intraventricular neuroendoscopic approaches and techniques by reconstituting natural CSF flow in a cadaveric model. In 10 fresh human cadavers, a simple cervical laminectomy and dural opening were made, and a 12-gauge arterial catheter was introduced. Saline was continuously perfused at physiological CSF pressures to reconstitute the subarachnoid space and ventricles. A neuroendoscope was subsequently inserted via a standard right frontal bur hole. In 8 of the 10 cadavers, adequate reconstitution and endoscopic access of the lateral and third ventricles were achieved. In 2 cadavers, ventricular access was not feasible, perhaps because of a small ventricle size and/or deteriorated tissue quality. In all 8 cadavers with successful CSF flow reconstitution and endoscopic access, identifying the foramen of Monro was possible, as was performing septum pellucidotomy and endoscopic third ventriculostomy. Furthermore, navigation of the cerebral aqueduct, fourth ventricle, prepontine cistern, and suprasellar cistern via the lamina terminalis was possible, providing a complementary educational paradigm for resident education that cannot typically be performed in live surgery. Surgical simulation plays a critical and increasingly prominent role in surgical education, particularly for techniques with steep learning curves including intraventricular neuroendoscopic procedures. This novel model provides feasible and realistic surgical simulation of neuroendoscopic intraventricular procedures and approaches.

http://thejns.org/doi/abs/10.3171/2014.10.JNS1497

KEY WORDS simulation; endoscopy; third ventriculostomy; surgical training; cadaver; cerebrospinal fluid; surgical technique

$\mathrm{S}$ URGICAL skills are traditionally developed in a purely clinical setting, with initial skill acquisition occurring on live patients in real time. This apprenticeship model has both major advantages and major disadvantages to patients and residents alike., ${ }^{9,17,18}$ In particular, when the surgical skills involved are characterized by a steep learning curve (for example, endoscopy or robotic surgery), prior experience with surgical equipment and rehearsed navigation of the operative anatomy are mandatory. Additional restraints placed on trainee exposure to operative cases throughout the training process may be a progressively limiting factor in education. For these reasons, surgical simulation in 3D virtual reality and livetissue models for trainee education has garnered consid- erable popularity in recent years. ${ }^{9,11,12,14}$ The ability to rehearse skills required to perform key steps in an operative procedure before performing live surgery, acquire basic familiarity with equipment and surgical approaches, and build the psychosomatic foundations required to perform a given operation may improve surgical training and directly transfer to surgical experience in the operating room. ${ }^{11,12,14}$

However, certain surgical procedures are particularly challenging to simulate and are limited by constraints in anatomical accuracy and the lack of haptic feedback. For example, neuroendoscopic approaches to intraventricular pathology are extremely difficult to model using only virtual reality. Although endoscopy is widely simulated, $, 2,2,4,711,15$ because of the anatomical complexity, varia-

ABBREVIATIONS ETV = endoscopic third ventriculostomy; FTDL = Fresh Tissue Dissection Laboratory; USC = University of Southern California. SUBMITTED January 24, 2014. ACCEPTED October 7, 2014.

INCLUDE WHEN CITING Published online April 10, 2015; DOI: 10.3171/2014.10.JNS1497.

DISCLOSURE The authors report no conflict of interest concerning the materials or methods used in this study or the findings specified in this paper. 
tion, and unique haptic feedback associated with intraventricular procedures, current virtual models lack a high degree of authenticity. For example, during an endoscopic third ventriculostomy (ETV), an advanced understanding of the anatomy is required to confirm which ventricle has been entered, and puncturing the floor of the third ventricle without disturbing the basilar artery and pons below is a skill that is arguably best practiced with the tactile feedback of live tissue. Even the most advanced surgical simulator currently lacks the haptic feedback and natural anatomical variation required to accurately facilitate the preoperative acquisition of neuroendoscopic skills. ${ }^{8}$

Despite the obvious advantages of a cadaveric model as pertains to real human tissue and normal anatomical variations, thus far it has been virtually impossible to simulate neuroendoscopic intraventricular approaches. Cadavers lack the circulating CSF system required for performing intraventricular neuroendoscopy, and to our knowledge no prior method of reconstituting ventricular CSF flow has been successfully developed and used for surgical training purposes. Tubbs et al. injected saline directly into the ventricles of cadavers, performing postmortem studies to establish whether ventricular dilation had been successful; however, they did not extend their study to the feasibility of endoscopic exploration and procedure simulation. ${ }^{16}$ The aim of the present feasibility study was to develop a working model of CSF dynamics using human cadavers that was sufficient to support the simulation of neuroendoscopic intraventricular approaches for surgical training purposes.

\section{Methods}

Ten fresh human cadavers were used to demonstrate the feasibility of the proposed model at the University of Southern California (USC) Fresh Tissue Dissection Laboratory (FTDL). The FTDL was created as a multidisciplinary educational tool for residents and medical students and operates under an internal review board-approved standard operating procedure established at our institution. All cadaver procurement and care follow strict guidelines and California state law. Cadavers were obtained within 7 to 21 days of death and were stored in crypts maintained at $3.3^{\circ}$ to $5.6^{\circ} \mathrm{C}$ until 2 hours before use, at which point they were initially placed prone. Neurosurgical residents at USC performed all procedures, including laminectomy and endoscope insertion. The trainee level ranged from postgraduate year 1 to 6 , and all procedures were done under the careful instruction of an attending neurosurgeon with an active practice in neuroendoscopy. In each specimen, a cervical laminectomy and 3-mm midline durotomy were performed, followed by the insertion of a 12-gauge pediatric arterial catheter into the intradural and/or subarachnoid space (Fig. 1). Following a watertight multilayer closure, the catheter was secured using multiple 0-silk sutures. Saline was manually injected into the catheter to ensure patent flow and then continuously infiltrated into the catheter via a Medtronic Bio-Pump (BP80, Medtronic SNT) to reconstitute the CSF space to a pressure of 15-30 $\mathrm{mm} \mathrm{Hg}$, as measured at the catheter site. Each specimen was then positioned supine, and a neurosurgical trainee was instructed to gain intraventricular access via a right

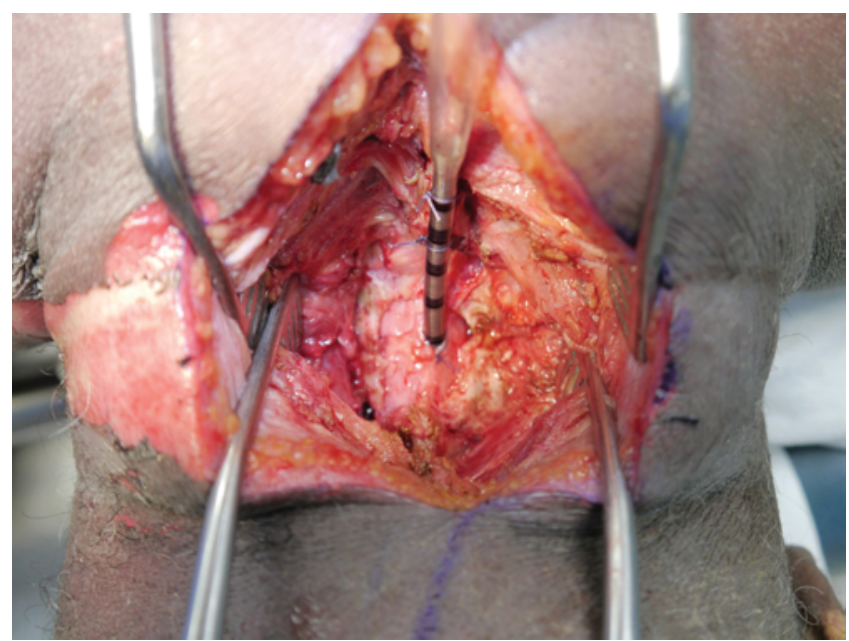

FIG. 1. Cervical laminectomy with durotomy and insertion of a pediatric arterial catheter into the intradural space. Normal saline was injected through the tube for the reconstitution of CSF into the ventricular space. Figure is available in color online only.

frontal bur hole approach (Fig. 2) using a trocar-based neuroendoscopic system with a high-definition monitor and recording capability (Karl Storz GmbH \& Co. KG). The initial goals were to identify normal lateral ventricular anatomy, navigate the foramen of Monro, and, when possible, perform an ETV and septum pellucidotomy. Additional exploration of the basilar cistern and/or fourth ventricle using $0^{\circ}$ and $30^{\circ}$ endoscopes was encouraged. Trainee confidence in the procedure was assessed via a self-reported questionnaire. The questionnaire evaluated self-reported gains on a Likert scale of 1-5. The categories were "gained new knowledge," "learned new techniques," "feel safer performing the procedure," "pre-procedure confidence," and "post-procedure confidence."

\section{Results}

Successful reconstitution of and access to the intraventricular system, defined as identification of normal lateral and third ventricular anatomical structures, were achieved in 8 of 10 specimens (Fig. 3). Although adequate "CSF" perfusion was confirmed in all 10 specimens by flow through the ventricular trocar, ventricular structures did not always maintain full integrity, probably because of variations in ventricle size and the tissue quality of the specimens. In 2 cadavers, endoscopic access could not be achieved because of a small ventricle size and/or poor brain integrity. Septum pellucidotomy was performed in all 8 cases in which adequate ventricular access had been achieved. Endoscopic third ventriculostomy was also performed in all 8 successful access cases (Figs. 4 and 5), permitting navigation and observation of interpeduncular and prepontine cistern anatomy. In all 8 of these cases, normal lateral ventricular and third ventricular anatomy was visualized, including the foramen of Monro, choroid plexus, septal vein, thalamostriate vein, lamina terminalis, tuber cinereum, cerebral aqueduct, mammillary bodies, and basilar artery. Although no injection of the vasculature was concurrently performed, the vessels in fresh cadavers maintained their natural coloring to some extent, thereby 


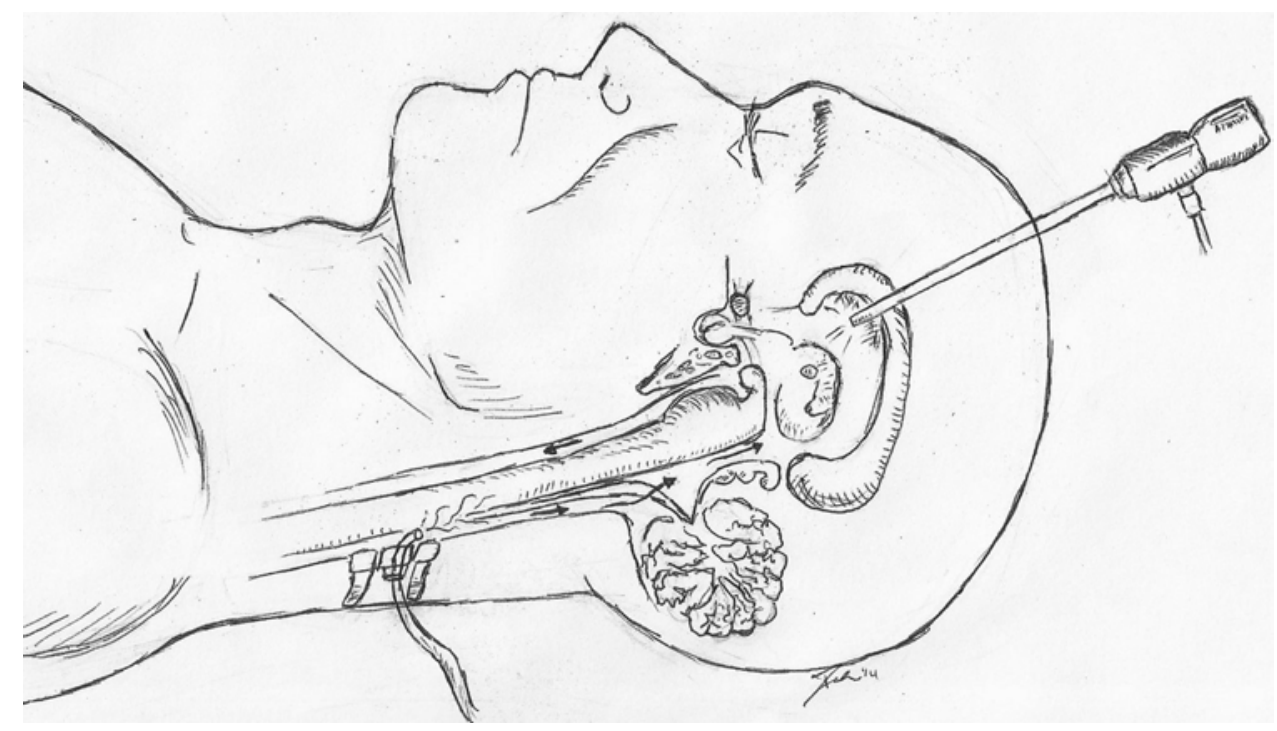

FIG. 2. Schematic of set-up. Following the laminectomy and pressurized injection of saline, the ventricular system was reconstituted and the endoscope was inserted. Visualization of the ventricles and surrounding structures was achieved, and various procedures, including an ETV and anatomical exploration, were successfully performed. Copyright Daniel R. Kramer. Published with permission.

facilitating identification of key vascular structures (for example, thalamostriate vein, septal vein, basilar artery). In 4 cases, cranial nerve, bony, and/or vascular anatomy of the prepontine, cerebellopontine, and cerebellomedullary cisterns was visualized (Fig. 6). In 2 cases, the cerebral aqueduct was navigated to visualize the fourth ventricular, dorsal brainstem, and cerebellar anatomy (Figs. 7 and 8).

Trainee confidence levels were assessed using a questionnaire to evaluate the experience. In the 8 cadavers that were successfully cannulated with CSF space reconstitution, 9 trainees participated and filled out questionnaires. On a Likert scale of $1-5$, the mean rating for each category was as follows: gained new knowledge, 4.7; learned new techniques, 4.6; feel safer performing the procedure, 4.6; pre-procedure confidence, 3.0 ; and post-procedure confidence, 4.2. Although not powered to objectively detect a difference between pre- and postprocedural confidence, all trainees reported an increase in postprocedure confidence.

\section{Discussion}

Over the past 2 decades, simulator-based surgical training models have been recognized as important and made a priority by major academic institutions. Although documentation of specific benchmarks in surgical skills is now required, concurrent limitations in trainee work hours may have contributed to constraints in operative skill acquisition during the surgical training process. To maximize the efficiency of time spent in the operating room during live surgery, simulation of operative procedures outside the operating room can get residents ahead on the learning curve for a surgical procedure. . $^{3,411,12,14}$ Our model is the first to simulate a variety of neuroendoscopic intraventricular procedures using a novel cadaveric CSF reconstitution model.

Given the recent success of surgical simulations s,4,11,12,14 $^{3,1}$ and the difficulty in adequately modeling intraventricular approaches with virtual reality, we described a feasible
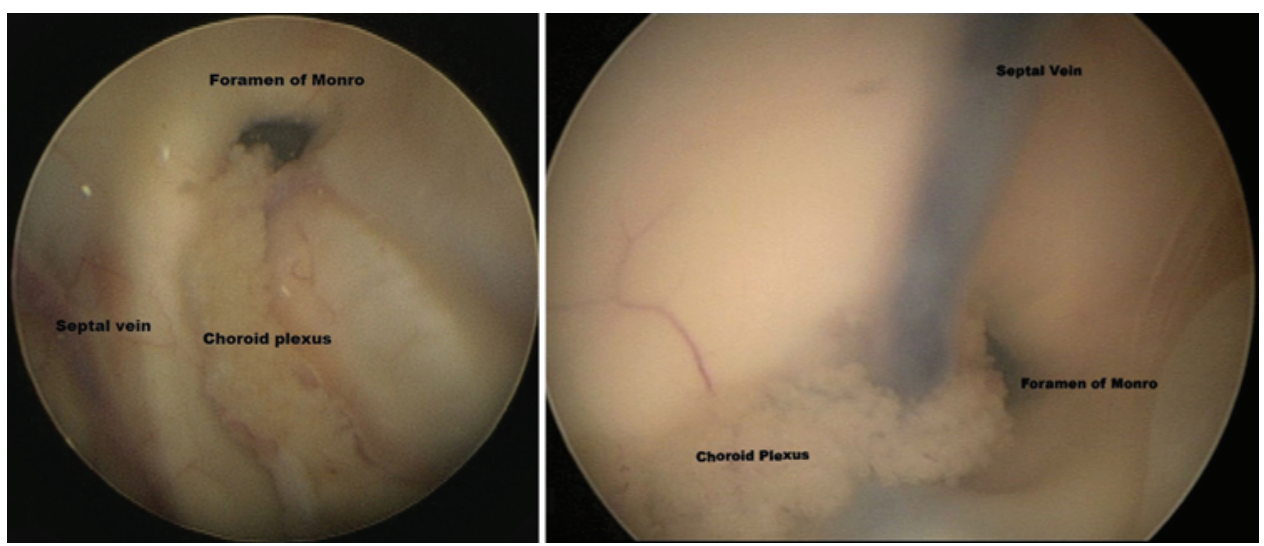

FIG. 3. The right lateral ventricle visualized endoscopically in 2 different cadavers (left and right) after infusion of the CSF space with saline. The septal vein and choroid plexus can be seen clearly in both, as can the foramen of Monro. Figure is available in color online only. 

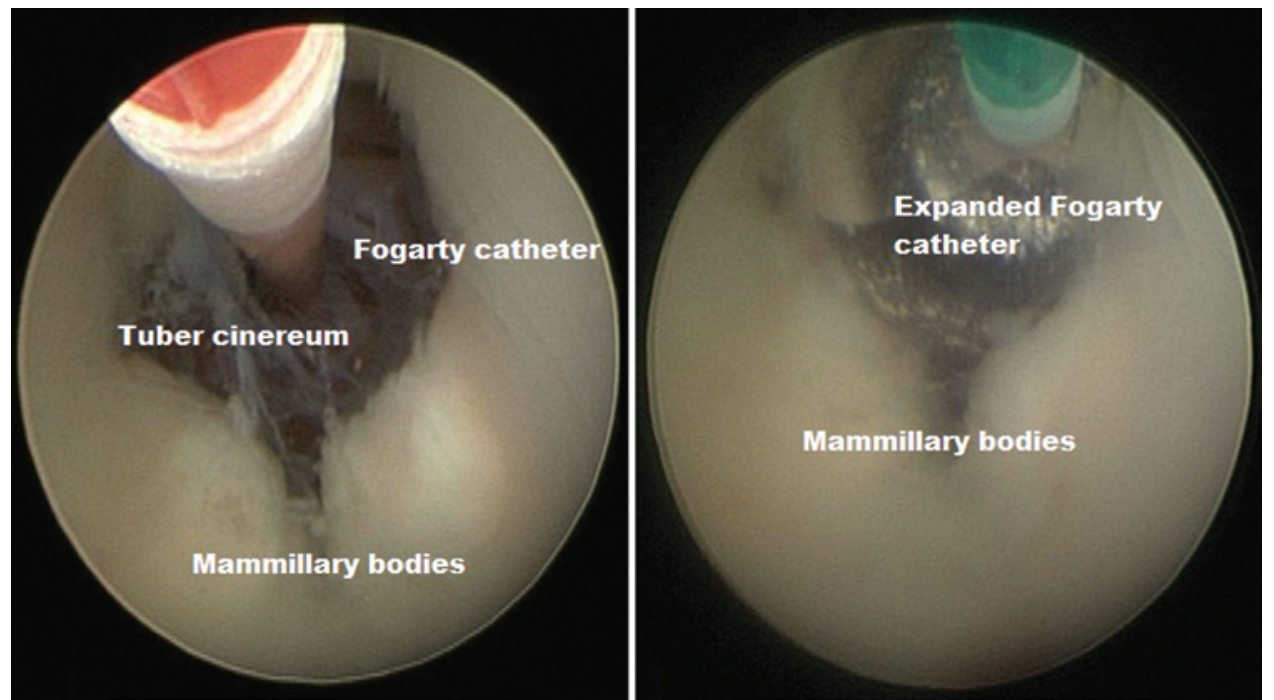

FIG. 4. Left: The floor of the third ventricle can be seen just prior to an ETV, after insertion of the Fogarty catheter. The mammillary bodies and the tuber cinereum can both be seen. Right: The balloon attached to the Fogarty catheter was expanded, opening the tuber cinereum. Figure is available in color online only.

model in which trainees can practice myriad neuroendoscopic procedures, including ETV, septum pellucidotomy, lamina terminalis fenestration, biopsy, and aqueductoplasty. Our model provides the benefits of true human anatomy, proper haptic feedback, and the opportunity to practice the procedure from skin incision to closure in the presence of lifelike CSF pressure and flow. Additionally, this model permits "neurotourism," allowing trainees to practice with different types of scopes, gain experience maneuvering the endoscope with the realistic feedback of disrupting surrounding structures, and explore anatomical structures that are normally not visualized from an approach to the lateral ventricle (for example, fourth ventricle and cerebellopontine angle). This method of CSF

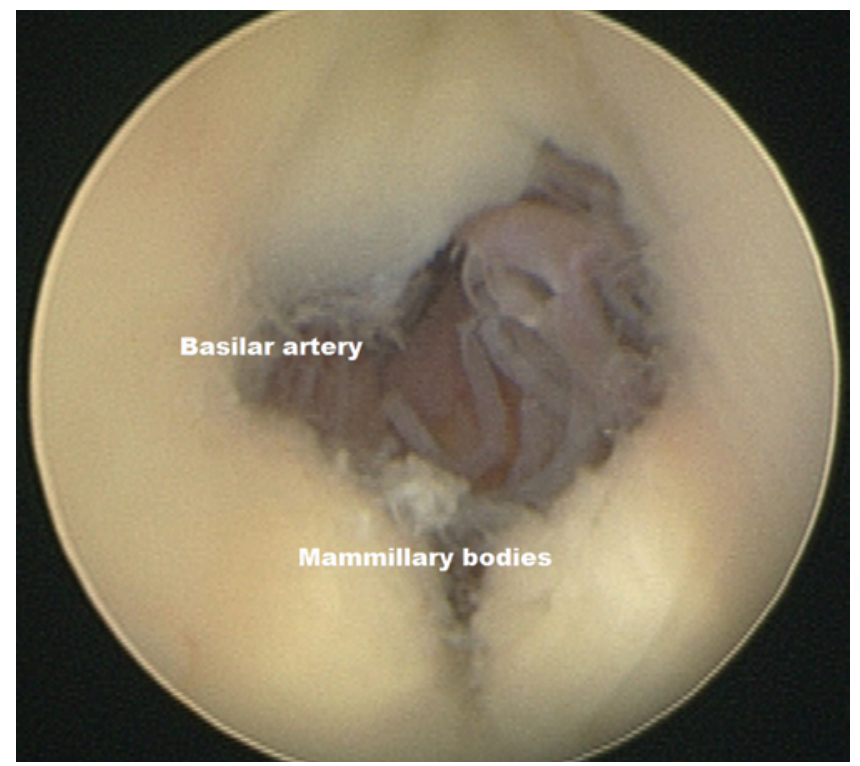

FIG. 5. The basilar artery was better seen through the floor of the third ventricle after a ventriculostomy was created. Figure is available in color online only. reconstitution is one that can be easily replicated with a relatively high degree of success, particularly in a fresh cadaver.

Although this novel technique is intended for trainee education, the recirculation of fluid through the ventricular system from the subarachnoid space in the spine sheds some light on the flow dynamics of CSF. Traditionally, early experiments in CSF dynamics have described flow up from the lumbar subarachnoid space through the cisterna magna, into the ventricular spaces. ${ }^{5,6,10,13}$ Significant active flow in the cranial direction was demonstrated with radioactive isotopes, ${ }^{10,13}$ more specifically, bidirectional flow, caudally in the posterior compartment and cranially in the anterior compartment, was reported. The flow demonstrated in the current model extended from the posterior compartment and reconstituted the entire CSF compartment. Although this is in opposition to the natural CSF flow, ${ }^{6,13}$ the pressurized flow in a nondynamic fluid system

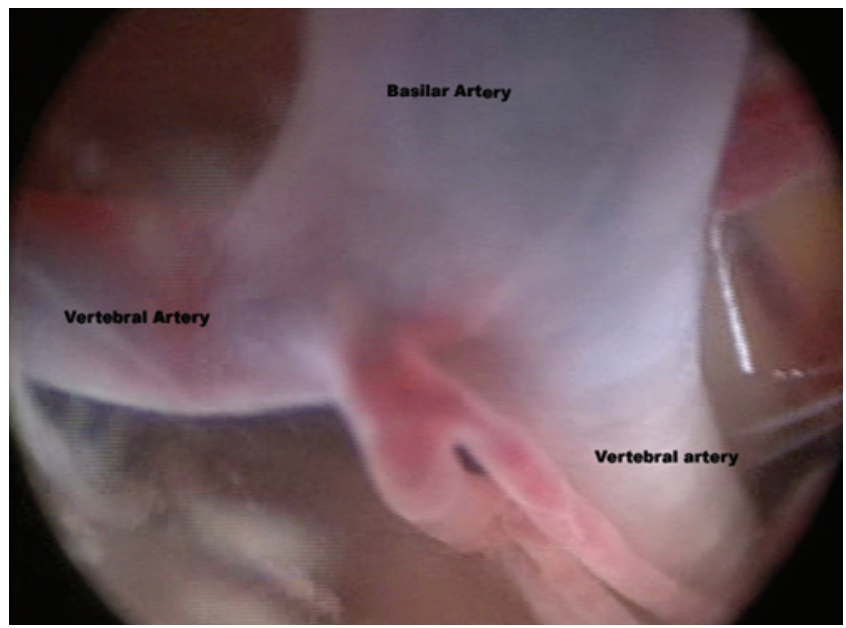

FIG. 6. Exploring further into the prepontine cistern, the basilar artery at its origin from the 2 vertebral arteries was noted. Figure is available in color online only. 


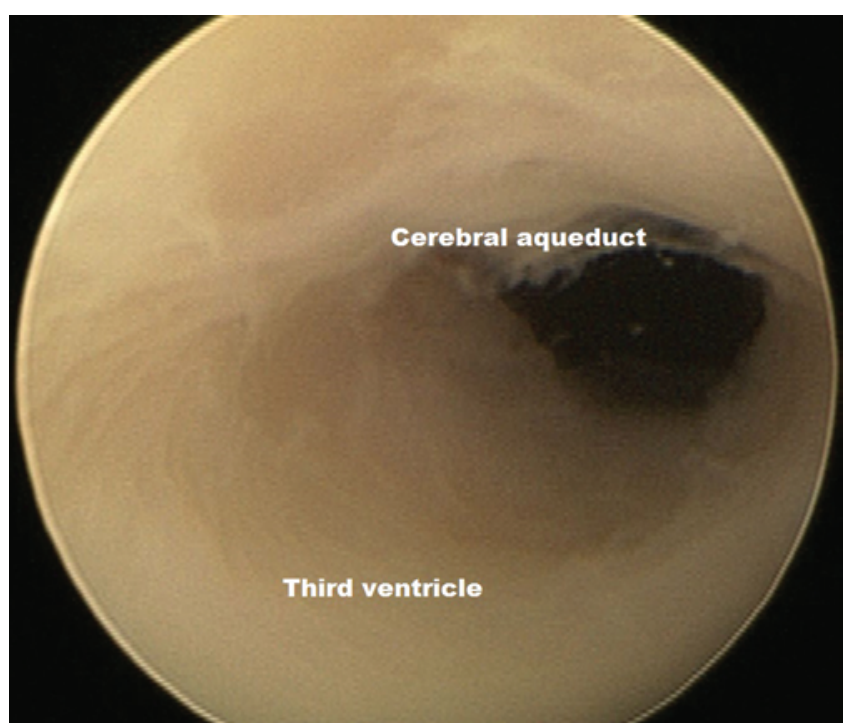

FIG. 7. A $30^{\circ}$ endoscope was inserted, and ventricular spaces were explored. Here the cerebral aqueduct was noted when looking back with the $30^{\circ}$ scope when inside the third ventricle. Figure is available in color online only.

appears to be easily reversed; if valves or other structural barriers exist to direct CSF flow, they are easily overcome at physiological pressures in the opposite direction. Injection into the ventricles or through the foramen of Magendie or Luschka is a more direct approach to reinstating CSF-like flow into the subarachnoid system and has been accomplished. ${ }^{16}$ However, the spine was found to be easier for 2 reasons: the simplicity of a laminectomy and spinal subarachnoid injection, and the relative preservation of the ventricular and basilar system. With regard to the second point, the goal of this technical exercise from a trainee standpoint was to provide training in endoscopic skills as well as anatomical study. Exploration of the fourth ventricle and the basilar system is not a standard approach from the lateral ventricles but was highly educational and readily feasible in this study.

Certain limitations to this model-in particular, its cost-may prevent it from being universally accepted by all training programs. The FTDL receives approximately 4 cadavers per week that are used for various surgical simulation experiences across a multitude of surgical specialties at USC. The Los Angeles County Office of Decedent Affairs takes responsibility for much of the cost of obtaining these specimens. Although this provides an ideal training scenario at USC, we realize that arrangements at other locations may be more costly. However, this model could feasibly be incorporated into neurosurgical training courses or other industry-sponsored educational sessions with a high degree of reproducibility and success. Since the ventricular system is relatively untouched in other models of neurosurgical procedures, this technique could be added to most cranial cadaveric training sessions. This method can also be used with only a cadaveric head provided that the inferior exit points of the subarachnoid space are sealed to allow adequate perfusion of the ventricular system. Nevertheless, obtaining the number of cadavers required to use this model to the extent of adequately pre-

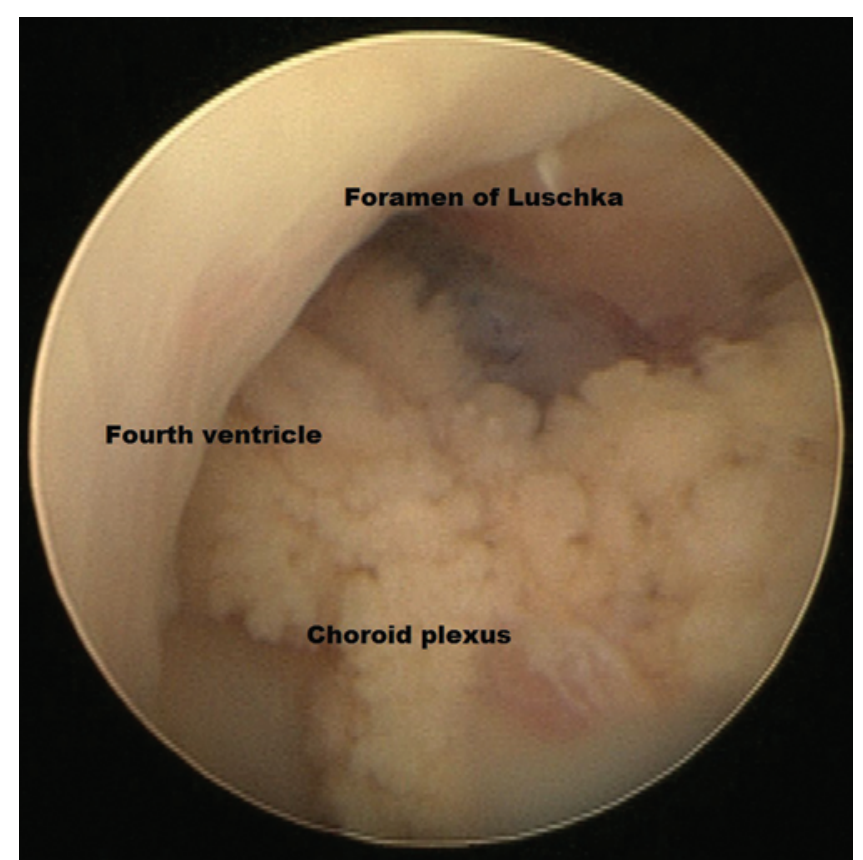

FIG. 8. The fourth ventricle was navigated in several cadavers. Here the foramen of Luschka can be seen as well as the choroid plexus. Figure is available in color online only.

paring all residents for proficiency in ETV and other intraventricular procedures would be impossible. This model is more useful as an adjunct to clinical experience, allowing the trainee to gain some experience prior to his or her time in the operating room.

Another limitation is the reliability of accessing and completing the intended neurosurgical procedure. Variations in anatomy and specimen age clearly limit one's ability to reliably access the cerebral ventricles. Hydrocephalus is a common feature of many intraventricular neuroendoscopic procedures, thereby facilitating navigation and visualization of intraventricular anatomy. In 2 of the specimens, we were unable to visualize the intraventricular anatomy. Each of these specimens had been obtained closer to 21 days after death, bringing into question the integrity of the cerebral tissue. Furthermore, in these 2 specimens, 2 passes were necessary to obtain "CSF" flow from the ventricle, suggesting either a small ventricle size or distorted anatomy or both. We observed that even after the successful attempts, the cerebral tissue did not always maintain its integrity, and we propose that the more transcerebral trocar passes required to access the ventricles, the less likely that the normal anatomy will maintain integrity adequate for navigation. Another limitation of the current study was the inability to augment these cadaveric dissections with preprocedural CT or MRI studies and the added benefits of neuronavigation. In the future, we hope to substantiate this feasibility study with improved imaging and instruments, as well as with prospective validation studies assessing the benefit(s) in surgical trainee psychomotor skills associated with neuroendoscopic procedures.

\section{Conclusions}

In this era of limitations on resident involvement in 
the operating room during complex approaches, surgical skills must be supplemented and validated by alternative simulation-based training paradigms prior to live surgical participation. The highest correlation with operative reality in surgical training is a cadaveric model. Our method of CSF reconstitution of the ventricular system is the first of its kind, allowing surgical training for neuroendoscopic intraventricular approaches. By continuously infusing the ventricles, trainees can reliably practice basic neuroendoscopic ventricular procedures including septum pellucidotomy, ETV, and others. The realistic anatomy, haptic feedback, and exposure to the same equipment used in live surgery make this cadaveric model ideal for resident training in neuroendoscopy. In the future, this type of simulation training may be incorporated into larger simulation sessions such as resident boot camp or annual meetings.

\section{References}

1. Bharathan R, Setchell T, Miskry T, Darzi A, Aggarwal R: Gynecological endoscopy skills training and assessment: a review. J Minim Invasive Gynecol 21:28-43, 2014

2. Caversaccio M, Eichenberger A, Häusler R: Virtual simulator as a training tool for endonasal surgery. Am J Rhinol 17:283-290, 2003

3. Davies J, Khatib M, Bello F: Open surgical simulation-a review. J Surg Educ 70:618-627, 2013

4. Dawe SR, Windsor JA, Broeders JA, Cregan PC, Hewett PJ, Maddern GJ: A systematic review of surgical skills transfer after simulation-based training: laparoscopic cholecystectomy and endoscopy. Ann Surg 259:236-248, 2014

5. Di Chiro G: Observations on the circulation of the cerebrospinal fluid. Acta Radiol Diagn (Stockh) 5:988-1002, 1966

6. Di Chiro G, Larson SM, Harrington T, Johnston GS, Green MV, Swann SJ: Descent of cerevrospinal fluid to spinal subarachnoid space. Acta Radiol Diagn (Stockh) 14:379-384, 1973

7. Edmond CV Jr: Impact of the endoscopic sinus surgical simulator on operating room performance. Laryngoscope 112: $1148-1158,2002$

8. Gasco J, Holbrook TJ, Patel A, Smith A, Paulson D, Muns A, et al: Neurosurgery simulation in residency training: feasibility, cost, and educational benefit. Neurosurgery 73 (Suppl 1):39-45, 2013

9. Gurusamy KS, Aggarwal R, Palanivelu L, Davidson BR: Virtual reality training for surgical trainees in laparoscopic surgery. Cochrane Database Syst Rev (1):CD006575, 2009

10. Rieselbach RE, Di Chiro G, Freireich EJ, Rall DP: Subarachnoid distribution of drugs after lumbar injection. N Engl J Med 267:1273-1278, 1962
11. Satava RM: Accomplishments and challenges of surgical simulation. Surg Endosc 15:232-241, 2001

12. Sturm LP, Windsor JA, Cosman PH, Cregan P, Hewett PJ, Maddern GJ: A systematic review of skills transfer after surgical simulation training. Ann Surg 248:166-179, 2008

13. Symss NP, Oi S: Theories of cerebrospinal fluid dynamics and hydrocephalus: historical trend. J Neurosurg Pediatr 11:170-177, 2013

14. Tan SS, Sarker SK: Simulation in surgery: a review. Scott Med J 56:104-109, 2011

15. Tolsdorff B, Pommert A, Höhne KH, Petersik A, Pflesser B, Tiede U, et al: Virtual reality: a new paranasal sinus surgery simulator. Laryngoscope 120:420-426, 2010

16. Tubbs RS, Loukas M, Shoja MM, Wellons JC, Cohen-Gadol AA: Feasibility of ventricular expansion postmortem: a novel laboratory model for neurosurgical training that simulates intraventricular endoscopic surgery. J Neurosurg 111:11651167,2009

17. van Det MJ, Meijerink WJ, Hoff C, Middel B, Pierie JP: Effective and efficient learning in the operating theater with intraoperative video-enhanced surgical procedure training. Surg Endosc 27:2947-2954, 2013

18. Willaert W, Van De Putte D, Van Renterghem K, Van Nieuwenhove Y, Ceelen W, Pattyn P: Training models in laparoscopy: a systematic review comparing their effectiveness in learning surgical skills. Acta Chir Belg 113:77-95, 2013

\section{Author Contributions}

Conception and design: Kramer, Winer, Robison, Minneti, Zada. Acquisition of data: Kramer, Winer, Robison, Ohiorhenuan, Minneti, Zada. Analysis and interpretation of data: Winer, Ohiorhenuan, Zada. Drafting the article: Kramer, Winer, Zada. Critically revising the article: Kramer, Winer, Ohiorhenuan, Giannotta, Zada. Reviewed submitted version of manuscript: Kramer, Winer, Robison, Ohiorhenuan, Giannotta, Zada. Approved the final version of the manuscript on behalf of all authors: Kramer. Administrative/technical/material support: Minneti. Study supervision: Giannotta, Zada.

\section{Supplemental Information}

\section{Previous Presentation}

Portions of this work were presented in poster form at the Congress of Neurological Surgeons Annual Meeting held in San Francisco, California, on October 19-23, 2013.

\section{Correspondence}

Daniel R. Kramer, 1200 N. State St., Ste. 3300, Los Angeles, CA 90033. email: dankramer29@gmail.com. 